\section{P1-439 EFFECT OF REGULAR EXERCISE ON THE NITRIC OXIDE LEVELS IN SALIVA}

doi:10.1136/jech.2011.142976g.29

${ }^{1} \mathrm{D}$ H Han, ${ }^{1}{ }^{1} \mathrm{H}$ S Shin, ${ }^{1} \mathrm{M}$ S Kim, ${ }^{2}$ W Kim, ${ }^{1} \mathrm{H}$ M Kim, ${ }^{1} \mathrm{H}$ D Kim. ${ }^{1}$ School of Dentistry, Seoul National University, Seoul, Republic of Korea; ${ }^{2}$ Health Care System, Gangnam Center, Seoul National University Hospital, Seoul, Republic of Korea

Introduction Nitric oxide (NO) regulates blood flow and both muscle contraction and metabolism during exercise. The plasma levels of NO increase after exercise. However, studies that determined the association between the levels of salivary $\mathrm{NO}$ and regular exercise are also lacking. The aim of this study was to assess the relationship of the levels of salivary $\mathrm{NO}$ with physical activity among Korean elderly.

Methods 176 subjects aged 48-84 years old were cross-sectionally surveyed. All participants underwent oral and general examination. Age, gender, education level, regular exercise, smoking were evaluated through interview. Periodontal health status was evaluated by clinical attachment loss over $6 \mathrm{~mm}$ (CAL6 $\mathrm{mm}$ ) for six points of 12 index teeth (total 72 sites). The number of CAL6 $\mathrm{mm}$ sites and the number of teeth were counted. Fasting plasma glucose level and blood pressure were measured. Unstimulated saliva was collected for 10 min. Salivary NO was determined by the Griess reagent. ANCOVA and Multiple linear regression analyses were applied.

Results After correcting for differences in for age, gender, the number of CAL6 $\mathrm{mm}$ sites, the number of teeth, $\mathrm{pH}$ in saliva, salivary flow rate, fasting plasma glucose level, systolic blood pressure and diastolic blood pressure, those who exercise everyday had the lowest salivary NO level (66.9 umol/l vs $104.3 \mathrm{umol} / \mathrm{l}$ and $121.3 \mathrm{umol} / \mathrm{l}, \mathrm{p}=0.082$ ), which is not significant. Salivary NO levels had a dose-effect relationship with the frequency of regular exercise ( $\beta=-13.5, p=0.022$ ).

Conclusion The results of the present study suggest that regular exercise may induce lower levels of NO.

\section{P1-440 LATENT CLASS ANALYSIS OF STUDENT SUBSTANCE USE}

doi:10.1136/jech.2011.142976g.30

${ }^{1}$ W J Harrison, ${ }^{*}{ }^{2} \mathrm{C}$ Montana, ${ }^{2} \mathrm{~B}$ M Bewick, ${ }^{1} \mathrm{M}$ S Gilthorpe, ${ }^{1,2} \mathrm{R}$ M West. ${ }^{1}$ Centre for Epidemiology \& Biostatistics, University of Leeds, Leeds, UK; ${ }^{2}$ Leeds Institute of Health Sciences, University of Leeds, Leeds, UK

Introduction Data were collected from a student population to investigate how substance use is related to perceived substance use of friends and the wider University population, during the period February to May 2009. The outcome measure was use of any of nine substances in the previous 3 months, giving differing patterns of use (trajectories) for each student. 4309 students were available for analysis.

Methods We used latent class analysis (LCA) to classify the students into trajectory subgroups and investigated how these trajectories were associated with covariates of interest. The emerging classes contained types of students rather than all individuals. Model fit was explored comparing log-likelihood statistics with consideration of model parsimony.

Results The model with two latent classes was preferred, containing one "low risk" class $(3313,90.6 \%)$ and one "high risk" class (344, $9.4 \%)$. Students in the "high risk" class perceived a higher proportion of others to be using more than the median amount of each substance, and had a higher mean knowledge score on a substance use quiz.

Conclusion The latent class structure was informative, with students well differentiated into the two substance use classes. We have thus identified factors that are associated with heavier substance use. While educational campaigns that employ "scare tactics" are unlikely to be successful, a campaign highlighting the disparity between perceived and measured levels of risk could be developed and be targeted towards the different classes of substance use seen here.

\section{P1-441 ESTIMATING THE FINANCIAL COST OF MURDER IN GLASGOW: THE MURDER CAPITAL OF WESTERN EUROPE}

doi:10.1136/jech.2011.142976g.31

${ }^{1} \mathrm{M}$ Harvey, ${ }^{*}{ }^{2} \mathrm{D}$ Williams, ${ }^{2} \mathrm{P}$ Donnelly. ' University of Minnesota, Minnesota, USA; ${ }^{2}$ University of St Andrews, St Andrews, Fife, UK

Introduction Compared to the rest of the UK, Scotland faces elevated levels of interpersonal violence. A large proportion of the violence is focused in Glasgow, which has acquired the label, Murder Capital of Western Europe. While violence is a leading burden on health, it also has a considerable financial impact. Until now, the cost of violence was based on dated estimates from England to Wales. This study estimates the financial cost of murder in Glasgow.

Method Data were supplied by the Scottish Violence Reduction Unit, on all murders in Glasgow between 2002 and 2009. The costing analysis considered four gross cost categories: lost output due to homicide (human capital), incarceration costs, investigation costs, and costs of physical and emotional impact.

Results Findings highlight a general reduction in the number of homicides between 2002 (50) and 2009 (29) with a peak in 2004 (62). Moreover, while overall costs have generally declined, the average cost of murder has increased between 2002 (£69.9M average $£ 1.39 \mathrm{M}$ ) and 2009 ( $£ 46.39 \mathrm{M}$, average $£ 1.60 \mathrm{M})$. The estimated total financial burden for murders committed in the 8-year period was in excess of $£ 523$ Million.

Conclusion The study suggests that previous figures have grossly underestimated the financial cost of murder in Glasgow and that the new estimates provide a more representative indication of the financial burden in Scotland. Moreover, the reducing murder rate coincides with the implementation of major violence reduction initiatives in the City. The findings therefore offer additional support for continued investment in Public Health initiatives to reduce violence in Scotland.

\section{P1-442 ANTENATAL MATERNAL FACTORS AND MILD COGNITIVE LIMITATIONS IN THE OFFSPRING}

doi:10.1136/jech.2011.142976g.32

${ }^{1,2}$ U Heikura, ${ }^{*} \mathrm{~A} L$ Hartikainen, ${ }^{1} \mathrm{~T}$ Norström, ${ }^{4,5} \mathrm{~A}$ Pouta, ${ }^{1,6} \mathrm{~A}$ Taanila, ${ }^{1,7} \mathrm{M}$ R Järvelin. ${ }^{1}$ Institute of Health Sciences, University of Oulu, Oulu, Finland; ${ }^{2}$ Verve, Oulu, Finland; ${ }^{3}$ Department of Clinical Sciences, Obstetrics and Gynecology, University of Oulu, Oulu, Finland; ${ }^{4}$ National Institute for Health and Welfare, Oulu, Finland; ${ }^{5}$ Department of Obstetrics and Gynecology, University Hospital, Oulu, Finland; ${ }^{6}$ Unit of General Practice, University Hospital, Oulu, Finland; 'Department of Epidemiology and Biostatistics, Imperial College London, School of Public Health, London, UK

Introduction There is a lack of epidemiological studies on early social and biological environmental factors, which may have an effect on development during childhood. The aim of this life-course cohort study is to explore the association between maternal gestational factors, by focusing on hypertensive disorders, and mild cognitive limitations (MCL, intelligence quotient, IQ 50-85) in the offspring. Study population and Methods An 11.5 year follow-up study of the Northern Finland Birth Cohort 1986 (NFBC 1986). The study included 8847 singleton children, whose IQ was not below 50. 198 children had MCL. Maternal gestational hypertension $(\mathrm{GH})$ was defined as de novo hypertension arising after mid-pregnancy in 